\title{
Position and field-dependent analysis of SNSPD waveforms (Conference Presentation), (Withdrawal Notice)
}

Claire Marvinney, Yun-Yi Pai, Brian Lerner, Matthew Feldman, Jie Zhang, et al.

Claire E. Marvinney, Yun-Yi Pai, Brian E. Lerner, Matthew A Feldman, Jie Zhang, Alexander A. Puretzky, Aaron J. Miller, Benjamin J. Lawrie, "Position and field-dependent analysis of SNSPD waveforms (Conference Presentation), (Withdrawal Notice)," Proc. SPIE 11721, Advanced Photon Counting Techniques XV, 117210H (10 May 2021); doi: 10.1117/12.2585925 


\section{Position and field-dependent analysis of SNSPD waveforms (Conference Presentation) (Withdrawal Notice)}

Claire E. Marvinney, Yun-Yi Pai, Brian E Lemer, J ie Zhang, Alexander A. Puretzky, and Benjamin J . Lawrie Oak Ridge National Lab. (United States)

Mathew A. Feldman,

Vanderbilt Univ. (United States)

Aaron J. Miller,

Quantum Opus, ШC (United States)

Proceedings Volume 11721, Advanced Photon Counting Techniques XV; $117210 \mathrm{H}$ (2021)

https:// doi.org/10.1117/12.2585925

Event SPIE Defense + Commercial Sensing, 2021, Online Only, United States

Online Public ation Date: 12 April 2021

Withdrawn from Publication: 7 May 2021

Publisher's Note: This presentation rec ording, orig inally published on 12 A pril 2021, was withdrawn per a uthor request. 